\title{
De l'art de chambarder la société de consommation
}

Portrait d'un récupérateur

Portrait of a Recycler or the Art of Turning Consumer Society Upside Down

\section{Violeta Ramirez}

\section{(2) OpenEdition}

Journals

Édition électronique

URL : https://journals.openedition.org/tc/8057

DOI : $10.4000 /$ tc. 8057

ISSN : 1952-420X

Éditeur

Éditions de l'EHESS

Édition imprimée

Date de publication : 31 octobre 2016

Pagination : 290-293

ISBN : 9782713225291

ISSN : 0248-6016

Référence électronique

Violeta Ramirez, « De l'art de chambarder la société de consommation », Techniques \& Culture [En ligne], 65-66 | 2016, mis en ligne le 31 octobre 2016, consulté le 29 septembre 2022. URL : http:// journals.openedition.org/tc/8057 ; DOI : https://doi.org/10.4000/tc.8057 


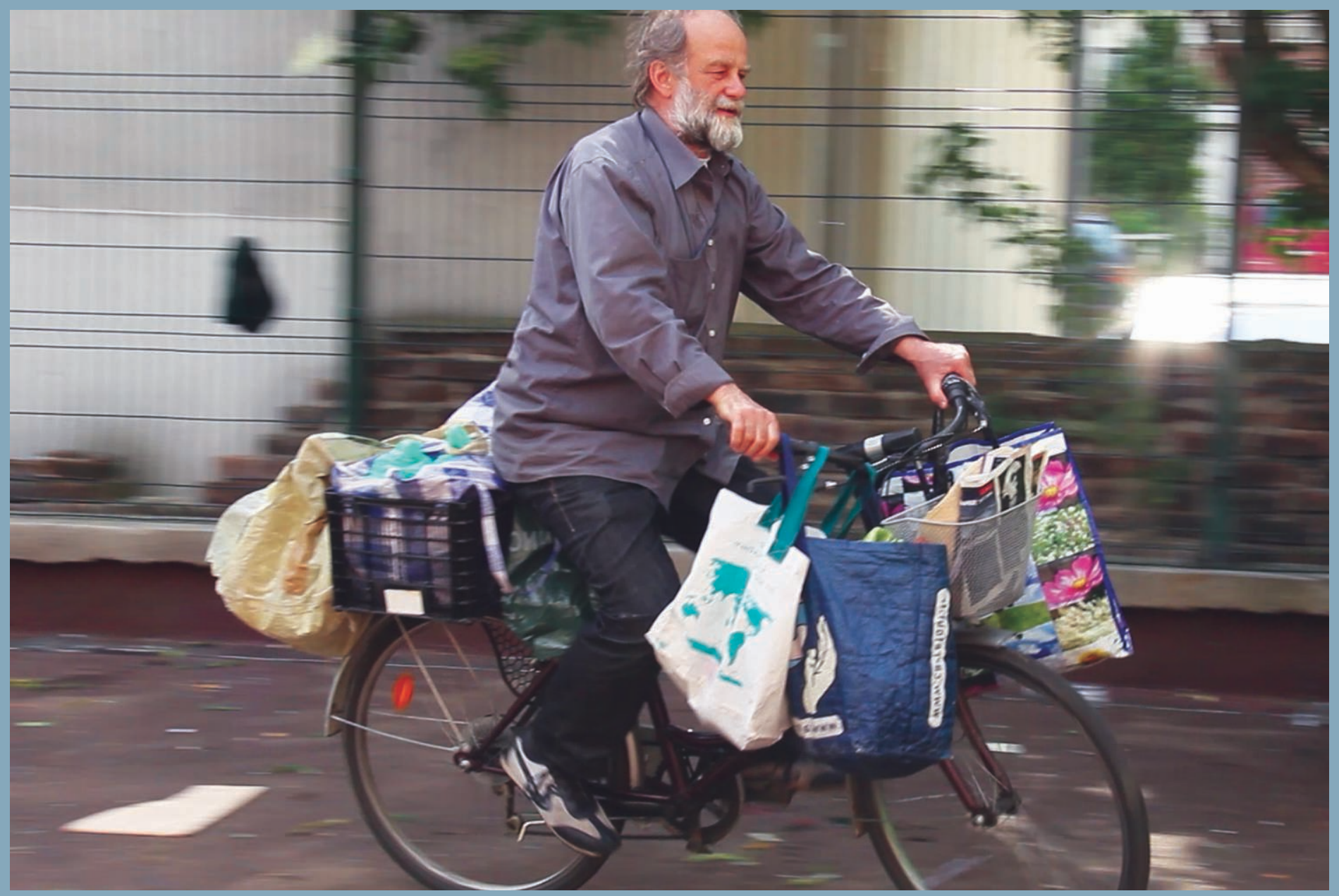




\section{De l'art de chambarder la société de consommation} Portrait d'un récupérateur

À travers les outils méthodologiques de l'anthropologie filmique, je me suis fixé comme objectif de suivre la chaîne de production et de récupération des déchets alimentaires dans un marché municipal en région parisienne. Dans le processus observé, les produits traités comme des déchets par les marchands sont requalifiés comme nourriture par les récupérateurs. Ainsi, ce phénomène montre combien la valeur attribuée aux objets et aux matières (en l'occurrence, à la nourriture) dépend du contexte, du milieu social et des régimes de valeur de chaque groupe.

Cette démarche de recyclage alimentaire chamboule les valeurs de la société de consommation dans laquelle nous vivons. Cette deuxième vie inattendue de la nourriture récupérée entraîne une remise en question de la logique marchande et des taux trop élevés de production et de gaspillage des sociétés contemporaines.

Daniel, le protagoniste de ce récit en images, a décidé de circuler au sein de cette économie des restes. Ancien économiste aujourd'hui à la retraite, il n'a pas économiquement besoin de récupérer dans les poubelles pour se nourrir. Sa participation à cette économie des marges relève d'un choix personnel et constitue un acte de résistance à la société de l'hyperconsommation.

Voilà maintenant cinq ans que Daniel a troqué son statut de client contre celui de récupérateur au marché alimentaire de la Place du 11 novembre à Malakoff, où il se rend trois fois par semaine. Vers 13 heures, lorsque le marché ferme ses portes au public, les marchands se défont des produits abîmés ou trop mûrs en les jetant dans les bennes à ordures roulantes. Ces poubelles sont ensuite amenées par les ripeurs à l'extérieur des halles, où leur contenu est évacué puis compacté dans deux grandes bennes vertes. C'est à ce moment-là que les récupérateurs interceptent les poubelles et procèdent à la récupération des fruits et légumes consommables qui jonchent le fond des bennes.

La zone du parking jouxtant les halles constitue donc l'espace de travail de différentes personnes. Tout d'abord, s'y affaire un groupe de ripeurs tamouls d'origine sri lankaise, 
tous employés municipaux responsables du nettoyage du marché et du fonctionnement des machines. Puis, on retrouve des récupérateurs de nourriture, parmi lesquels Daniel et un groupe de femmes d'origine maghrébine. Ces trois profils de personnes fréquentent cet espace pour des raisons diverses et variées (les unes par nécessité, les autres en tant que travailleurs salariés, et Daniel, par engagement militant). Malgré ces différences, elles organisent un espace commun de travail en partageant le territoire, les ressources et certaines tâches.

L'absence de conflit manifeste lors de mes observations est en grande part dû au rôle d'intermédiaire que Daniel s'est donné dans cette organisation de travail. En ce qui concerne le traitement des ordures, Daniel travaille comme «assistant» des ripeurs afin de les aider à déplacer les bennes roulantes et à séparer et compacter les cartons dans la machine verte réservée à cet usage. En se chargeant de ces tâches, il économise du temps de travail aux employés municipaux; en échange les récupérateurs sont laissés libres de fouiller dans les poubelles. Quant au partage de la ressource récupérée, il se fait en fonction des besoins des uns et des autres. N'étant pas nécessiteux, Daniel laisse les autres se servir en priorité; lui dispose du reste.

Au long du déroulement des activités, on observe des moments de travail collaboratif et des moments de labeur individuel. La fouille des poubelles constitue un travail solitaire: chacun face à sa benne, les yeux rivés sur les déchets, se laisse surprendre par ce que les poubelles lui offrent. En amont et en aval de ce moment, l'organisation de l'espace de travail et le partage de la nourriture demandent la coopération des personnes présentes.

Après la fouille, afin d'éviter l'acheminement de produits en mauvais état, il est impératif de trier les trouvailles sur place. Pour cela, le toucher et la dégustation sont nécessaires, mais le poids et le volume des aliments doivent savoir également être appréciés.

La récolte est souvent généreuse en rapport au besoin des glaneurs et à leur capacité de transport. Là réside l'enjeu fondamental de la démarche des récupérateurs: quoi faire du surplus de la ressource trouvée dans les poubelles. Nous constatons que les récupérateurs éprouvent un malaise au moment d'abandonner les trouvailles et de les (re)définir comme déchets.

Par souci de sobriété énergétique, Daniel ne conçoit pas d'emprunter un autre moyen de transport que le vélo pour acheminer la marchandise du marché à chez lui. Il a une voiture dont il se sert moins pour ses déplacements que pour constituer une sorte de grand garde-manger où stocker la nourriture qu'il lui reste à redistribuer.

Au bout de la chaîne de récupération, Daniel a trouvé des personnes intéressées par ces produits récupérés. Il a donc créé un circuit de distribution parmi lequel on retrouve ses voisines de l'immeuble, des amis et des personnes en situation d'exclusion qu'il croise dans la rue. Dans ce circuit de distribution alternatif, les produits sont livrés par Daniel en tant que dons, et reçus (ou refusés) comme tels par les destinataires. Avec cette action de récupération et de distribution, Daniel transforme la valeur marchandise des aliments et instaure, parmi un cercle réduit de personnes, une économie alternative du don. 


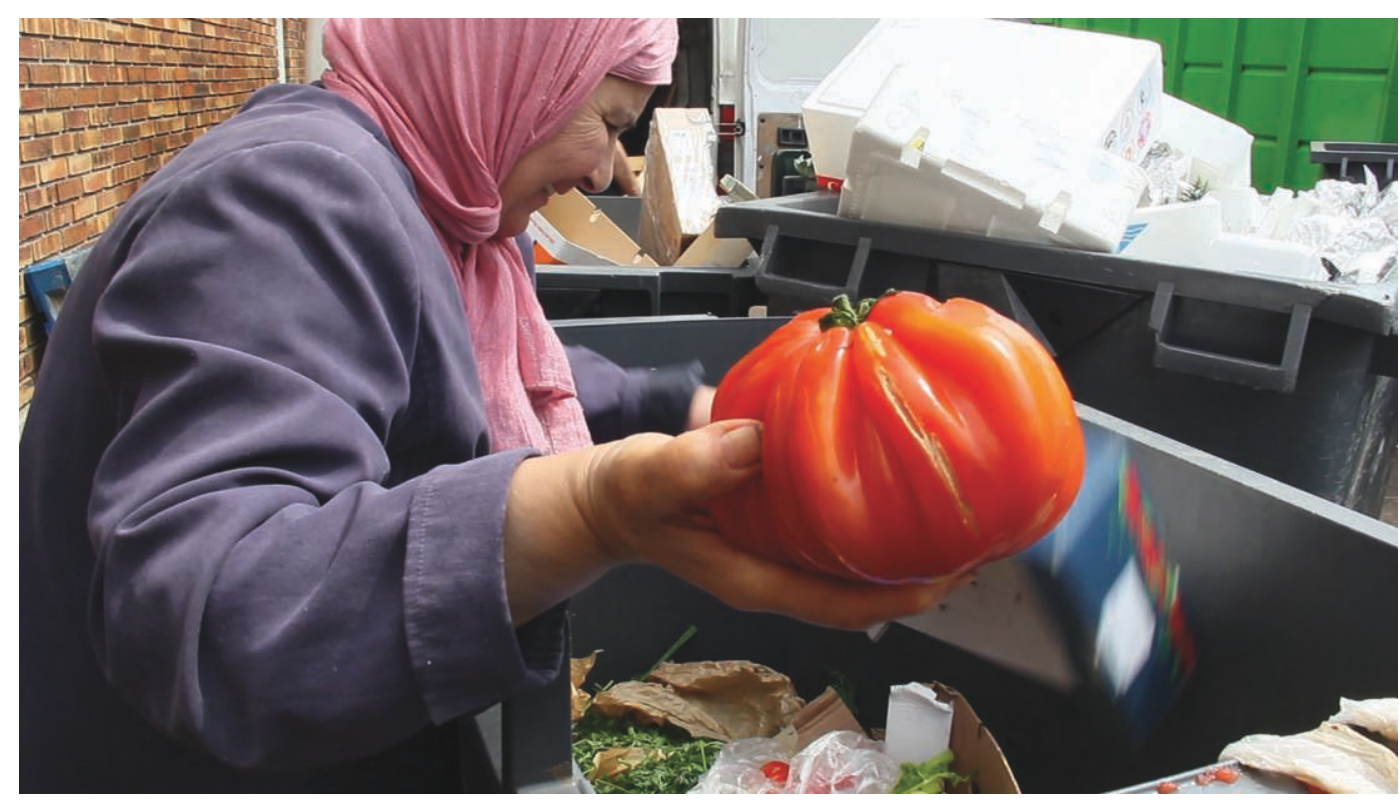

La chasse aux trésors

\section{En ligne}

Retrouvez l'article complet sur revues.org, TechniquesECulture 65-66 « Réparer le monde. Excès, reste et innovation »: http://tc.revues.org.

\section{I'auteure}

Violeta Ramírez effectue une thèse en anthropologie filmique au sein du laboratoire «Histoire des Arts et des Représentations» (HAR) de l'université Paris Ouest Nanterre La Défense. Ses recherches portent sur les modes de vie durables et la sobriété énergétique en France. Visionnez le film documentaire Le Récupérateur (40 mn), ainsi que les travaux de Violeta Ramírez sur son site internet: www.violetaramirez.com.

\section{Iconographie}

Crédits photographiques pour l'ensemble des images. (C) Violeta Ramírez

\section{Pour citer cet article}

Ramírez, V. 2016 «De l'art de chambarder la société de consommation. Portrait d'un récupérateur. », Techniques ECulture 65-66 «Réparer le monde. Excès, reste et innovation», p. 290-293. 